\title{
Analyses of Power Output of Piezoelectric Energy Harvesting Devices Directly Connected to a Resistive Load Using a Coupled Piezoelectric-Circuit Finite Element Method
}

\author{
Meiling Zhu ${ }^{1 *}$, Emma Worthington ${ }^{1}$ and James Njuguna ${ }^{2}$ \\ ${ }^{1}$ Department of Materials, ${ }^{2}$ Department of Sustainable Systems \\ Cranfield University, Bedfordshire, MK43 0AL UK
}

\begin{abstract}
A coupled piezoelectric-circuit finite element model (CPC-FEM) is proposed for the first time to study the power output of a vibration-based piezoelectric vibration-based piezoelectric energy harvesting devices (EHDs) that is directly connected to a resistive load. Special focus is given to the effect of the resistive load value on the vibrational amplitude of the piezoelectric EHDs, and thus on the current, voltage, and power generated by the EHDs. In the literature, these outputs are widely assumed to be independent of the resistive load value for the reduction in complexity of modelling and simulation. The presented CPC-FEM uses a cantilever with sandwich structure and a seismic mass attached to the tip to study the following load characteristics of the EHD as a result of changing the resistive load value: (1) the electric outputs of the EHD: current through and voltage across the resistive load, (2) the power dissipated by the resistive load, (3) the vibration amplitude of tip displacement of the cantilever, and (4) the shift in resonant frequency of the cantilever. Investigation results shows significant dependences of the vibration characteristics of the piezoelectric EHDs on the externally connected resistive load are found, rather than independence as previously assumed in most literature.
\end{abstract}

The CPC-FEM is capable of predicting the generated power output of the EHDs with different resistive load value while simultaneously calculating the effect of the resistive load value on the vibration amplitude. The CPC-FEM is invaluable for validating the performance

\footnotetext{
* Corresponding author's address: Building 70, Department of Materials, Cranfield University, Bedfordshire, MK43 0AL UK. Tel.: +44 (01234) 750111 ext. 2092; Fax: +44 (01234) 751346; E-mail address: m.zhu@cranfield.ac.uk.
} 
of designed EHDs before fabrication and testing, thereby reducing the recurring costs associated with repeat fabrication and trials. In addition, the proposed CPC-FEM is potentially useful in device designs optimisations for maximal power generation.

Keywords: finite element method (FEM), vibration-based piezoelectric energy harvesting devices (EHDs), coupled piezoelectric-circuit (CPC), load characteristics 


\section{Introduction}

With technology advancements over the last few decades, the vast reduction in size and power consumption of complementary metal-oxide-semiconductor (CMOS) circuitry has led to a great research effort toward energy harvesting devices (EHDs) for the development of wireless sensors and ubiquitous wireless networks of communication nodes [1-5]. Significant progress has been made and a large number of vibration-based EHDs have been proposed and tested using various mechanisms, including electromagnetic, electro-static and piezoelectric [2-6]. Piezoelectric EHDs have received special attention due to their self-contained power without requiring an external voltage source, highest energy density, and good dynamic responses, and ability to scavenge energy in the range of $1-200 \mu \mathrm{W} / \mathrm{cm}^{3}$ from ambient vibration energy sources $[5,7]$ (possibly higher in the nearby future once optimized design and improved piezoelectric materials are available). This energy level is suitable for power demand in the range of an average consumption of $100 \mu \mathrm{W}$ [8], hence particularly suitable for wireless sensors and communication nodes.

In particular, cantilever-based piezoelectric EHDs with a seismic mass and sandwich structure are an attractive geometry for harvesting energy from vibration. This mainly owe to the fact that such a structure is designed to work in the first bending mode with a lower stiffness, offers a lower resonant frequency, which can be easily matched to the target ambient vibration frequency for maximum power generation.

The power output of EHDs dictates device suitability for most applications. As such, the evaluation of the power output of an EHD for a given excitation frequency and a given amplitude of ambient vibration plays an important role in the design of EHDs. Availability of a proper model that can be used to calculate the power output for designs is crucial.

According to modal analysis theory, the governing equations of piezoelectric EHDs can be written as follows: 


$$
\begin{gathered}
M \ddot{z}(t)+C \dot{z}(t)+K z(t)=M \ddot{y}(t)-\Theta V_{p}(t) \\
\Theta z(t)-C_{p} V_{p}(t)=Q_{p}(t)
\end{gathered}
$$

where: $M, C$ and $K$ represent the effective mechanical mass, mechanical damping and mechanical stiffness respectively; $\Theta$ represents the effective electromechanical coupling coefficient of a piezoelectric structure; $V_{p}(t)$ and $Q_{p}(t)$ represent the voltage across the piezoelectric electrodes and the electric charge generated on the electrodes due to the ambient vibration, respectively; $z(t)$ is the mechanical displacement of mass, relative to the fixed point of the cantilever; $\ddot{y}(t)$ is the acceleration at the fixed point of the device from the ambient vibration environment; and $C_{p}$ is the electric capacitance of a piezoelectric structure. Piezoelectric EHDs are often connected with an electric circuit to transform the harvested electric energy into a usable form [9-12]. The circuit may include: a rectifier to convert AC to DC, a super-capacitor to store harvested energy, an inductive load to boost the voltage output of the transducer, or a resistive load to be used to characterize the capability of the designed EHDs in power output. Most existing modelling techniques assume the vibration amplitude of the EHDs to be independent of the connected circuit, however, the piezoelectric EHD's output voltage and current are greatly influenced by the circuit connected. The question to address is: how does the connected circuit influences the vibration response, and in turn the power output, of a piezoelectric EHD? At the present state of knowledge, this question is unanswered in the literature for EHDs with an attached circuit. This paper therefore studies the effect of the resistive load value on the power output of the piezoelectric EHDs.

General equations for the electric outputs of a piezoelectric EHD directly connected with a electric resistor are

$$
\begin{aligned}
& V_{R}(t)=I_{R}(t) R \\
& P_{R}(t)=V_{R}(t) I_{R}(t)
\end{aligned}
$$


where: $V_{R}(t)$ represents the voltage across the resistive load; $I_{R}(t)$ the current through the resistive load; and $P_{R}(t)$ the power dissipated in the resistive load, which is a good indication of the actual power generated by the piezoelectric EHD.

For the configuration where a piezoelectric EHD is directly connected with a load resistor, the relationships between the output of the piezoelectric EHD and the input to the resistive load are

$$
\begin{aligned}
& V_{p}(t)=V_{R}(t) \\
& I_{R}(t)=\omega Q_{p}(t)
\end{aligned}
$$

From (1)-(6), it is observed that $z(t), V_{R}(t)$ and $I_{R}(t)$ (and therefore $P_{R}(t)$ ) are coupled together through $R$ and $\Theta$. This means that $z(t), V_{R}(t)$ and $I_{R}(t)$ are all affected by $R$ and $\Theta$. The present paper proposes a coupled piezoelectric-circuit finite element model (CPC-FEM) to study the power output, particularly focusing on the influence of the resistive load value on the vibration amplitude (the mechanical displacement) of the beam, therefore electric outputs of current, voltage, and power. The effects of electromechanical coupling on the vibration, and electric current, voltage and power are all simulated using the developed CPC-FEM. The purpose of this paper is to develop an understanding of how an externally connected electric load affects the power related output. Firstly, existing modelling techniques are briefly reviewed. A coupled piezoelectric-circuit FEM is then developed, in which piezoelectric EHDs are directly connected with an external electric circuit to calculate the electric outputs (current, voltage, and power), the tip displacement, and the resonant frequency shift. This is the first time in the literature CPC-FEM has been developed to perform such calculations.

\section{An overview of existing modelling methods}

A variety of modelling approaches have been used to analyse the output of the piezoelectric 
EHDs. Among them, uncoupled analyses, equivalent electric circuit methods, and advanced modelling methods have been proposed. In the followings, an overview of the existing methods and their existing deficiencies is given followed by the justification for the need of the proposed CPC-FEM.

\subsection{Uncoupled analyses}

Piezoelectric EHDs are complicated electromechanical dynamic systems. For simplicity, such devices have frequently been modelled as a current source in parallel with the piezoelectric capacitance $C_{p}[7-13]$. The assumption made in the method is that the vibration amplitude of the piezoelectric structure is independent of the impedance of the externally connected load. This means that the externally connected load has no influence on the amplitude of the vibration displacement. Reverting to the governing equations (1 and 2) of the piezoelectric EHDs, it can be easily seen that the assumption has ignored the coupling term $\Theta V_{R}$ on the right hand side of (1), reducing it to (7)

$$
M \ddot{z}(t)+C \dot{z}(t)+K z(t)=M \ddot{y}(t)
$$

Equation (7) represents an uncoupled system between electric and mechanical responses. This assumption is fairly reasonable in the case of extremely low coupling for some sensor analyses. However, piezoelectric EHDs use a high electromechanical coupling material to convert mechanical energy to electric energy, so they are an exceptional case, and the assumption is not valid. This is because the generated electric energy in turn influences mechanical vibrations through the high electromechanical coupling term, $\Theta V_{R}$, on the right hand side of (1). This means that the harvested electric energy (voltage across/current through the resistor) from the ambient vibration will reduce the structural vibration when the devices harvests energy, and this in turn reduces the harvested energy due to the reduced structural vibration. The evaluation of the coupling effects on the electrical and mechanical output of a 
piezoelectric EHD is an important task that requires attention. This paper proposes CPC-FEM to address this deficiency in coupled analyses methods.

\subsection{Equivalent electric circuit methods}

Stand alone piezoelectric sensors without connection to an external circuit are normally modelled as a short circuit where current source is in parallel with the piezoelectric capacitance $C_{p}$, or as an open circuit where voltage source is in series with $C_{p}$. The modelling method is mainly based on piezoelectric constitutive equations:

$$
\begin{aligned}
& T_{i}=c_{i j} S_{i}-e_{3 i} E_{3} \\
& D_{3}=e_{3 i} S_{i}+\varepsilon_{3}^{S} E_{3}
\end{aligned}
$$

In these equations, $T_{i}, S_{i}$, and $c_{i j}$ denote in turn components of the stress and strain vectors, and components of stiffness matrices measured at a constant electrical field; $E_{3}$ and $D_{3}$ are the $z$-components of the electric field and displacement; and $e_{3 i}$ and $\varepsilon_{3}^{S}$ the piezoelectric stress coefficient and the $z$-component of dielectric coefficient measured at a constant strain. For the calculation of the current source, it is assumed that $E_{3}=0$ in (8) and (9). One can then calculate the electric charge generated in the electrode surfaces and the electric current flowing through the electrodes based on $D_{3}=e_{3 i} S_{i}$. The vibration is calculated based on $T_{i}=c_{i j} S_{i}$ as the case calculated is $E_{3}=0$. For the calculation of a voltage source, a similar approach as the current source is used, that is, it is assumed that $D_{3}=0$ in (9). One can then calculate the electric voltage across piezoelectric electrodes based on $E_{3}=-e_{3 i} S_{i} / \varepsilon_{3}{ }^{s}$. It is obvious that the above calculations for charge or voltage are valid only in the case of $E_{3}=0$ or $D_{3}=0$. For piezoelectric EHDs connected with an external electric circuit, using the above equivalent electric circuit theory to calculate currents or voltages of the devices is not very 
well suited, as the EHD is not in the case of $E_{3}=0$ or $D_{3}=0$. Calculation of currents and voltages by using the above methods would lead to an over-estimation in power.

\subsection{Advanced modelling methods}

A few advanced models to analyze piezoelectric EHDs have also been reported in the literature. For example, Guyomar et al. [12] proposed a new approach for the case of high coupling to estimate the average harvested power. In their approach, the assumption made was that the external force $M \ddot{y}(t)$ and the velocity of the mass $\dot{z}(t)$ are in phase. This assumption is fairly reasonable for non-piezoelectric mechanical structures operating around resonance with low damping. Whether this assumption still hold for the case of high electromechanical coupling is questionable and has not been addressed in Ref. [12] report. Shu and Lien [7] developed an analytical model of power output for piezoelectric harvesting systems focusing on AC-DC output. Their research concluded that the harvested power depends on the input vibration characteristics (frequency and acceleration, the mass of generator, the electric load, the natural frequency, the mechanical damping ratio and the electromechanical coupling coefficient of system). Further analytical modelling and analysis of micro piezoelectric power generators was reported by $\mathrm{Lu}$ [14] who focused on the influence of load resistance on the output power of cantilevered piezoelectric bimorph.

It is not difficult to find that all the advanced modelling and simulation mentioned above are reliant on the availability of parameters of $M, K, C$ and $\Theta$. These parameters vary in different structures; subtle changes in geometry and mass location will change the parameters. Derivation of each individually designed structure would be a very time-consuming task. FEA is an advanced and reliable numerical modelling method, which has already been used to calculate sensors and actuators in numerous applications, and also used to calculate voltages in the open circuit and currents in the short circuit for piezoelectric EHDs [14]. However, 
FEA has not been used to predict the power output of piezoelectric EHDs that are connected to an external electric circuit, possibly because a suitable FEA model has not been put forward to date due to the complexity in analyses. This paper will present a CPC-FEM to study the effects of a resistive load on the vibration and also on the electric outputs: voltage, current and power, of the EHD.

\section{CPC-FEM Modelling and Simulations}

Commercially available FEA software (ANSYS Inc. Canonsburg, PA) [15], is utilized to develop the CPC numerical model of the piezoelectric EHD connected directly with a resistive load. Special focus is given to the analysis of power related outputs and vibrational characteristics, where the 'power related outputs' represents the current through and voltage across the resistor and the power dissipated in it, and the 'vibrational characteristics' represents the tip displacement and the resonant frequency shift of the piezoelectric EHD.

\subsection{Modelling Configuration}

The modelled geometry of a piezoelectric cantilever beam with a seismic mass is shown in Figure 1. The beam is a piezoelectric sandwich structure with a central brass substrate layer and two piezoelectric material layers, one each bonded to the top and bottom of the brass layer. The two piezoelectric layers can be wired in series so that their individual voltages add together to feed into an externally connected circuit, as shown in Figure 2(a), or in parallel so that their individual currents add together to feed into a connected resistor, as shown in Figure 2(b). In series, the two piezoelectric layers are oppositely polarized and the output terminals are the outside electrodes of the piezoelectric layers. In this case, an electric potential $\left(2 V_{p}\right)$ exists between the output terminals when the structure is subjected to a vibration at the fixed end of the beam. In parallel, the two piezoelectric layers are polarized in the same direction, 
and the outside electrodes are joined together to make one terminal. The central layer constitutes the other terminal. In this configuration, the same amount of charge exists on each outside electrode when the structure is subjected to vibration at the fixed end. These two configurations: parallel and series, were each studied for the comparison of the EHD outputs. In the developed finite element models (FEMs), the direction of polarization of the piezoelectric material is represented by the sign of the piezoelectric coefficients $e_{31}$ and $e_{33}$ [15]. For example, in series, the polarization of the bottom piezoelectric layer is opposite to that of top piezoelectric layer, shown in Figure 2(a), so $e_{31}$ and $e_{33}$ have opposite signs in the defined material property to the top piezoelectric material in the developed model. The electrode connections have been made by using the 'couple' commands, creating two common nodes for the piezoelectric EHD to fulfil the required connection. The external connected circuit is then connected with these two common nodes in the model. The location of the circuit with respect to the device can be arbitrary as it does not affect the analytical results. As the adhesives between the substrate and piezoelectric layers are very thin, the adhesive thicknesses are ignored in all simulations.

\subsection{CPC-FEM}

Figure 3 shows the developed CPC-FEM: a sandwich cantilever beam with piezoelectric layers on the top and bottom, and a seismic mass attached to the tip, where the electrodes of piezoelectric layers are connected to a load resistor. The 8-node, hexahedral, coupled-field element SOLID5 is used for the piezoelectric material, and the 8-node, linear, structural element SOLID45 for the non-piezoelectric material. To achieve this coupled piezoelectriccircuit analysis, the piezoelectric circuit element (CIRCU94) is connected with the piezoelectric element (SOLID5). CIRCU94 can model resistors, inductors, capacitors, current sources, or voltage sources. In the model, CIRCU94 is used to model the load resistor to study 
the power related outputs and vibrational characteristics. The resistive load value is defined by a real constant in the developed programme. The CIRCU94 resistor element is connected to the EHD through defined coupled common nodes, and the detailed connections are based on either the series or parallel configuration, shown in Figure 2(a) or (b).

The modelled geometric parameters and material properties are listed in Table 1, where all the geometric dimensions are purposely fixed and the resistive load value is variable to enable a focus of analyses on the effect of different resistive load value on the power related outputs and vibrational characteristics. The piezoelectric material chosen is PSI-5H4E from Piezo Systems, Inc. This material has a very high piezoelectric constant, and devices made with it are able to convert energy from the ambient vibration environment into more electric energy since the higher the piezoelectric constant, the higher the conversion of mechanical energy into electric energy. In the model, dielectric material loss is ignored but the piezoelectric material's mechanical loss (based on the piezoelectric material quality factor) is introduced into the model by use of the constant damping ratio $\xi_{\mathrm{p}}$, which is the ratio between the actual damping and critical damping, and is obtained from the relationship $\mathrm{Q}=\frac{1}{2 \xi_{p}}$ (the $Q$ value is 32 for the selected piezoelectric material). As the piezoelectric material data sheet gives the compliances, the elastic stiffness constants required in the model are obtained from the conversion of the compliances into the stiffness constants [17-19]. In the model, the substrate is made of brass and the seismic mass is tungsten-alloy for generating more power due to the higher density that is proportional to the generated power. For simulation, to simulate an ambient vibration environment, a constant vibrational amplitude of displacement of $3.96 \mu \mathrm{m}$ is applied to the fixed end of the cantilever. Harmonic analyses are first performed with different resistive load value. The electric current, voltage and power output of the EHD are extracted from the output data of SOLID5 and CIRCU94. The tip-displacements and resonant frequency shift are also extracted from the simulation results. The results are shown in 
Figures 4-7 and Tables 2-3.

\section{Results and discussions}

\subsection{Frequency responses of configuration in parallel}

Figure 4(a) shows the simulated frequency responses of the current and voltage outputs of the EHD (piezoelectric layers in parallel configuration) with the following listed resistive load values: (1) short circuit, where $R=10^{-20}$ in the developed CPC-FEM as it is impossible to let $R=0$ perform the calculation of $I_{R}=V_{R} / R ;(2)$ open circuit, where $R=10^{20}$, again, it is impossible to let $R=\infty$; and (3) a circuit connected with a resistor $R_{o p t}$, where $R_{o p t}=\frac{1}{\omega_{n} C_{p}}$ ( $\omega_{n}$ being the resonant frequency of the EHD). It can be seen from Figure 4(a) that for $R=0$, the piezoelectric EHD is a current source and $V_{R}=0$ (not shown in Figure 4(a) as $\left.V_{R}=0\right)$. All the current generated stays in the piezoelectric capacitor $C_{p}$, thus there is no electric current feeding into the output terminals and so the output power from the EHD is 0 , i.e., $P_{\text {out }}=0$. Similarly, for $R=\infty$, the piezoelectric EHD is a voltage source and $I_{P}=0$ (not shown in Figure 4(a) as $I_{R}=0$ ), the output power from the EHD is 0 , as $I_{R}=0$, i.e., $P_{\text {out }}=0$. But, for $R_{\text {opt }}=\frac{1}{\omega_{n} C_{p}}$, it can be seen that power is generated by the piezoelectric EHD, as there is current flowing through, and voltage developed across, the connected load resistor.

Figure 4(b) shows the simulated frequency responses of the dissipated power and tipdisplacement of the piezoelectric EHD with the same resistive load values as those for Figure 4(a). Here, the tip-displacement is greatly affected by the connected resistive load, as for $R=0 \rightarrow R_{o p t} \rightarrow \infty$, the amplitude at the resonant frequency decreases from $169 \mu \mathrm{m}$ to 
$71 \mu \mathrm{m}$ and then increases to $178 \mu \mathrm{m}$; the resonant frequency shifts from $78.5 \mathrm{~Hz}$ to $82 \mathrm{~Hz}$, and then to $83 \mathrm{~Hz}$; and the power output at the resonant frequency changes from $0 \mu \mathrm{W}$ to $60.6 \mu \mathrm{W}$ and then back to $0 \mu \mathrm{W}$. The connected resistive load has an obvious damping effect on the vibrational amplitudes, but it is not the case that the larger the resistive load, the higher the damping effect; it is more complicated than simple viscous damping. However, it can be concluded that the connected resistor has a significant effect on the vibrational amplitude of the piezoelectric EHD, and on the current, voltage and power in turn. Therefore, the coupling effect of the connected resistive load on the vibration characteristics needs to be considered for correct analysis of the piezoelectric EHD and its power output.

\subsection{Frequency responses of the configuration in series}

Figures 5(a) and (b) show the simulated frequency responses of the current and voltage, dissipated power and tip-displacement of the EHD (piezoelectric layers in series configuration) with similar but not same resistive load values as those for Figures 4(a) and (b) due to different $C_{p}$ for series configuration (the details is given below). Similar conclusions for piezoelectric layers in series can be obtained as for those in parallel.

Comparing Figure 4(a) with 5(a), and Figure 4(b) with 5(b), Tables 2 and 3 summarize the characteristics of the EHDs of piezoelectric layers in parallel and in series configuration for different resistive load value. It can be seen that for the parallel configuration, the electric capacitance of the EHD is four times as high as for the series configuration as the equation of the capacitance of the piezoelectric EHDs is

$$
C_{p}=n \frac{\varepsilon_{0} \varepsilon_{r} A}{h_{p}} \text { where }\left\{\begin{array}{l}
\text { in series : } n=0.5 \\
\text { in parallel }: n=2
\end{array}\right.
$$

where $h_{p}$ and $A$ are the thickness and electrode area of piezoelectric layers. As the 
capacitances are different, these lead to $R_{\text {opt } \rightarrow \text { series }}=4 R_{\text {opt } \rightarrow \text { parallel }}$ as

$$
R_{o p t}=\frac{1}{\omega_{n} C_{p}}
$$

It is worthwhile mentioning that here the optimised resistance is based on the assumption that the vibrational amplitude is independent of the externally connected resistor [1].

It is interesting to find that the different configuration of piezoelectric layers does not change the open and short circuit resonant frequencies $\left(f_{o c}\right.$ and $\left.f_{s c}\right)$, and so the generalized electromechanical coupling coefficient $\left(k_{31-E D H}\right)$ of the piezoelectric EHDs remains the same for different configuration of piezoelectric layers as $k_{31}$ is determined by the open and short circuit resonant frequencies [20, 21]:

$$
k_{31 \rightarrow E H D}^{2}=\frac{f_{o c}^{2}-f_{s c}^{2}}{f_{s c}^{2}}
$$

where $f_{o c}$ and $f_{s c}$ are the open circuit and short circuit resonant frequencies. For the designed piezoelectric EHD, $k_{31-E D H}=0.32$, which is lower than $k_{31}=0.44$ of the piezoelectric material itself. So it can be concluded that the introduced substrate has reduced $k_{31-E H D}$ of the structure. It should be borne in mind that $k_{31-E D H}$ of the structure is not the same as $k_{31}$ of the material and readers need to distinguish them at the design stage.

Furthermore, it can be observed that there are clear relationships of the current, voltage and output power between in parallel and in series configurations:

$$
\begin{aligned}
& V_{R \rightarrow \text { series }} \approx 2 V_{R \rightarrow \text { parallel }} \\
& I_{R \rightarrow \text { series }} \approx \frac{1}{2} V_{R \rightarrow \text { parallel }} \\
& P_{R \rightarrow \text { series }} \approx P_{R \rightarrow \text { parallel }} \\
& z_{\text {series }} \approx z_{\text {parallel }}
\end{aligned}
$$

It is also interesting to find that the different configuration changes the distribution of the current and voltage at the output terminals but does not significantly affect the overall power 
output and vibrational amplitude.

\subsection{Load characteristics}

To further evaluate the effect of the connected resistive load value on the performance of the piezoelectric EHDs, Figures 6(a), (b) and (c) show the currents through and voltages across and dissipated powers by the resistor, the displacements at the tip of the beam, and the resonant frequency shift versus a range of electric resistive load values for the parallel configuration. The current and voltage at maximum power output versus resistive load value are indicated in Figure 6(a) and the corresponding vibrational amplitude and power are also indicated in Figure 6(b). Similar conclusions for the load characteristics can be obtained as those for the simulated frequency responses, that is, (1) the current, voltage, and power dissipated depend significantly on the value of the load resistance, and (2) the value of the load resistance also significantly affects the vibrational characteristics of a piezoelectric EHD, including the vibrational amplitude and the resonant frequency. Furthermore, from Figure 6(b), some important phenomena can be observed: (1) the maximum power output of piezoelectric EHDs does not appear at the maximum vibrational displacement, and also (2) the maximum power output does not appear at the $R_{\text {opt }}$, as normally presented in literature. The reason for this is the possibly that the model proposed here has taken into account the vibrational amplitude that is affected by the externally connected resistance while the literature has to date ignored this crucial effect.

Figure 7(a) shows the relation of voltage versus current for the configuration of parallel connection of piezoelectric layers of EHDs, with a range of resistive load values to further describe the load characteristics of piezoelectric EHDs. The area with the hatched pattern in Figure 7(a) represents the output power (as the output power is the product of electric current and the electric voltage). The larger the area of the hatched pattern, the higher the power 
output. As the areas are 0 for $R=0$ and $R=\infty$ at both ends of the curve, it is obvious that an optimized electric resistance exists for maximum power generation. Figure 7(b) shows the relation of power versus vibrational amplitude of the piezoelectric EHD for the resistive load value as those for Figure 7(a). Figure 7(b) gives a better indication of the phenomena observed from Figure 6(b).

\section{Conclusions}

A CPC-FEM is, for the first time, proposed to study the power output of a vibration-based piezoelectric EHD that is directly connected to a resistive load. The effect of the resistive load value on the vibration amplitude of the EHD, and therefore on the current, voltage and power generated by the EHD, are investigated. These outputs are normally assumed to be independent of the resistive load value for the reduction in complexity of modelling and simulation. The studied CPC-FEM of the piezoelectric EHD is based on a cantilever with sandwich structure and a mass attached at the tip. The load characteristics, including (1) the electric current and voltage outputs, (2) the power dissipated by the connected resistive load, (3) the tip vibration displacement amplitude, and (4) the resonant frequency shift of the cantilever versus an externally connected resistive load, are all simulated using the developed CPC-FEM. Significant dependences of the vibration characteristics of the piezoelectric EHDs on the externally connected resistive load are found, rather than independence. One interesting and important result found from the analyses is that the maximum power output of piezoelectric EHDs does not appear at the maximum vibrational displacement, as the power is determined by the product of electric current and electric voltage.

In addition, the configurations with series and parallel connections of piezoelectric layers have been studied. It is found that the configurations can change the distribution of the electric current and voltage at the output terminals but cannot significantly change the power 
output and vibrational amplitude when the structure is subjected to same excitations.

At present, the results are based on the finite element method and further work is underway to experimentally support present FEA results. A testing bed will be built to verify experimentally all of the numerical results in our group. Although the present model cannot be completely confirmed by experimental results at the moment, the presented CPC-FEM has indeed shown the ability to calculate the generated power output and responded vibration, and frequency shift of vibration-based piezoelectric EHDs for a range of resistive load values. The CPC-FEM is therefore invaluable for validating the performance of designed devices and optimizing geometric parameters of piezoelectric EHDs before fabrication and testing, reducing cost recurring at design and trial stages.

It is worthwhile to mention that although the presented work is based on a piezoelectric EHD directly connected with a resistive load, the CPC-FEM is applicable to the analyses of piezoelectric EHDs directly connected with inductors, or capacitors, or independent current sources, or independent voltage sources and even their combinations.

\section{Acknowledgement}

The authors gratefully acknowledge the financial support from IMRC (the Cranfield Innovative Manufacturing Research Centre) via EPSRC (Engineering and Physics Science of Research Council in the UK) entitled "Design and Manufacturing of a Novel EHDs for Self Powered Sensors and ICT Applications" (IMRC-137), and platform programme (EP/D506638/1) entitled "Nanoscale Multifunctional Ferro-Materials and Devices" from EPSRC. 


\section{References}

1. S. Roundy, P. K. Wright and J. Rabaey, "A study of low level vibrations as a power source for wireless sensor nodes," Comput. Commun., vol. 26, pp. 1131-1144, 2003.

2. P. D. Mitchson, E. M. Yeatman, G. K. Rao, A. S. Holmes, and T.C. Green, "Energy harvesting from Human and machine motion for wireless electronic devcies," Proceeding of the IEEE, vol.96, No.9, pp. 1457-1486, September, 2008.

3. C. Kompis and S. Aliwell, "Energy harvesting technologies to enable romote and wireless sensing," Sensors and Instrumentation-Knowledge Transfer Network, June 2008.

4. S. Priya, "Advances in energy harvesting using low profile piezoelectric transducerds", Journal of Electroceramics, 19, 165-182, March 2007.

5. Spies, P., Pollak, M. and Rohmer, G., "Power management for energy harvesting applications", available on line:

http://www.iis.fraunhofer.de/Images/power_management_for_energy_harvesting_applicat ions_tcm278-91133.pdf (Accessed 13 ${ }^{\text {th }}$ Mar. 2009).

6. S. P. Beeby, M. J. Tudor and N. M. White, "Energy harvesting vibration sources for microsystems applications," Measur. Sci. Technol., vol. 17, pp. 175, 2006.

7. Y. C. Shu and I. C. Lien, "Analysis of power output for piezoelectric energy harvesting systems," Smart Mater Struct, vol. 15, pp. 1499, 2006.

8. S. Roundy, E. S. Leland, J. Baker, E. Carleton, E. Reilly, E. Lai, B. Otis, J. M. Rabaey, P. K. Wright and V. Sundararajan, "Improving power output for vibration-based energy scavengers," Pervasive Comput., IEEE, vol. 4, pp. 28-36, 2005.

9. E. Lefeuvre, A. Badel, C. Richard and D. Guyomar, "Piezoelectric energy harvesting devices optimization by synchronous electric charge extraction," Journal of Intelligent Material Systems and Structures, 16-Oct, 865-876, 2005

10. E. Lefeuvre, A. Badel, C. Richard, L. Petit, D. Guyomar, "A comparison between several vibration-powered piezoelectric generators for standalone systems," Sensors and Actuators A, 126, 405-416, 2006.

11. G. A. Lesieutre, G. K. Ottman and H. F. Hofmann, "Damping as a result of piezoelectric energy harvesting, Journal of Sound and Vibration, 269, 991-1001, 2004.

12. D. Guyomar, A. Badel, E. Lefeuvre and C. Richard, "Toward energy harvesting using active materials and conversion improvement by nonlinear processing," Ultrasonics, Ferroelectr. Freq. Contr., IEEE Trans, vol. 52, pp. 584-595, 2005.

13. G. K. Ottman, H. F. Hofmann and G. A. Lesieutre, "Optimized piezoelectric energy harvesting circuit using step-down converter in discontinuous conduction mode," Power Electr., IEEE Trans, vol. 18, pp. 696-703, 2003.

14. F. Lu, H. P. Lee and S. P. Lim, "Modelling and analysis of micropiezoelectric power 
generators for micro-electromechanical-systems applications," Smart Mater Struct, vol. 13, pp. 57-63, 2004.

15. ANSYS, "ANSYS Release 11.0 Documentation,"

16. S. Roundy, P. K. Wright and J. M. Rabaey, Energy Scavenging for Wireless Sensor Networks: With Special Focus on Vibrations. Springer, 2004.

17. M. Zhu, P. Kirby and M. Y. Lim, "Lagrange's formalism for modeling of a triaxial microaccelerometer with piezoelectric thin-film sensing," Sensors J, IEEE, vol. 4, pp. 455-463, 2004.

18. M. Zhu and G. Leighton, "Dimensional reduction study of piezoelectric ceramics constitutive equations from 3-D to 2-D and 1-D," Ultrasonics, Ferroelectr. Freq. Contr., IEEE Trans, vol. 54, pp. 584-595, 2008.

19. J. M. Whitney, Structural Analysis of Laminated Anisotropic Plates. CRC Press, 1987,

20. C. J. Cross and S. Fleeter, "Shunted pizoelectrics for passive control of turbomachine blading flow-induced vibration," Smart materials and Structures, 11, 239-248, April 2002 .

21. L. R. Corr and W. W. Clark, "Comparison of low-frequency piezoelectric switching shunt techniques for structural damping," Smart materials and Structures, 11, 370-376, May 2002. 


\section{List of Tables}

Table 1 Input geometric and material parameters used in the developed CPC-FEM

Table 2 Characteristics of piezoelectric layers in parallel and in series

Table 3 Comparison of the outputs for configurations in parallel and series 
Table 1 Input geometric and material parameters used in the developed CPC-FEM

\begin{tabular}{|c|c|}
\hline \multicolumn{2}{|c|}{ Substrate material: brass } \\
\hline Young's modulus (GPa) & 110 \\
\hline Poisson's ratio $\mu_{\mathrm{s}}$ & 0.23 \\
\hline Density $\left(\mathrm{kg} / \mathrm{m}^{3}\right)$ & 2330 \\
\hline $\begin{array}{l}\text { Length* } \times \text { width } \times \text { thickness }(\mathrm{mm}) \\
* \text { The total length of mass and } \\
\text { piezoelectric layer }\end{array}$ & $28 * \times 3.2 \times 0.1016$ \\
\hline \multicolumn{2}{|c|}{ Piezoelectric material: PSI-5H4E from Piezo Systems, Inc. } \\
\hline Young's modulus (GPa) & \\
\hline $\mathrm{E}_{11}$ & 62 \\
\hline $\mathrm{E}_{33}$ & 50 \\
\hline Poission's ratio & 0.3 \\
\hline Elastic constants:(GPa) & \\
\hline $\mathrm{C}_{11}$ & 110.8 \\
\hline $\mathrm{C}_{12}$ & 49.8 \\
\hline $\mathrm{C}_{13}$ & 49.8 \\
\hline $\mathrm{C}_{33}$ & 110.8 \\
\hline $\mathrm{C}_{44}$ & 30.5 \\
\hline Density $\rho_{\mathrm{p}}\left(\mathrm{kg} / \mathrm{m}^{3}\right)$ & 7800 \\
\hline $\begin{array}{l}\text { Piezoelectric constants }\left(\times 10^{-12} \mathrm{~m} / \text { volt }\right) \\
\qquad \mathrm{d}_{33} \\
\mathrm{~d}_{31}\end{array}$ & $\begin{array}{l}650 \\
-320\end{array}$ \\
\hline \multicolumn{2}{|l|}{ Coupling coefficients } \\
\hline $\mathrm{k}_{33}$ & 0.75 \\
\hline $\mathrm{k}_{31}$ & 0.44 \\
\hline Relative dielectric constant $\varepsilon_{33}$ & 3800 \\
\hline Mechanical quality factor Q & 32 \\
\hline Length $\times$ width $\times$ thickness $(\mathrm{mm})$ & $10.7 \times 3.2 \times 0.278$ \\
\hline \multicolumn{2}{|l|}{ Seismic mass: Tungsten } \\
\hline Young's modulus (GPa) & 400 \\
\hline Poisson's ratio $\mu_{\mathrm{s}}$ & 0.28 \\
\hline Density $\left(\mathrm{kg} / \mathrm{m}^{3}\right)$ & 17000 \\
\hline Length $\times$ width $\times$ thickness $(\mathrm{mm})$ & $17.3 \times 3.6 \times 7.7$ \\
\hline
\end{tabular}


Table 2 Characteristics of piezoelectric layers in parallel and in series

\begin{tabular}{|l|l|l|}
\hline Piezoelectric configuration & In parallel & In series \\
\hline Inherent electric capacitance $(\mathrm{nF}): C_{p}$ & 8.287 & 2.071 \\
\hline Open circuit resonant frequency $(\mathrm{Hz}): f_{o c}$ & 82.5 & 82.5 \\
\hline Short circuit resonant frequency $(\mathrm{Hz}): f_{s c}$ & 78.5 & 78.5 \\
\hline $\begin{array}{l}\text { Generalized electromechanical coupling } \\
\text { coefficient of the piezoelectric EHD: } \\
k_{31 \rightarrow E H D}^{2}\left(k_{31 \rightarrow E H D}\right) \text { 0.1045 (0.3233) }\end{array}$ & $0.1045(0.3233)$ \\
\hline Optimized resistance $(\mathrm{k} \Omega): R_{o p t}^{*}$ & 234.2 & 936.8 \\
\hline
\end{tabular}

* optimized resistance based on Ref. [1]. 
Table 3 Comparison of the outputs for configurations in parallel and series

\begin{tabular}{|c|c|c|c|}
\hline \multirow[t]{2}{*}{ Resistances } & \multirow[t]{2}{*}{ Outputs } & \multicolumn{2}{|c|}{ Piezoelectric layer configuration } \\
\hline & & In Parallel & In series \\
\hline \multirow[t]{4}{*}{$R=0$} & $I_{R-\max }(\mu \mathrm{A})$ & 140 & 70 \\
\hline & $V_{R-\max }(\mathrm{Volt})$ & 0 & 0 \\
\hline & $P_{R-\max }(\mu \mathrm{W})$ & 0 & 0 \\
\hline & $z_{\max }(\mu \mathrm{m})$ & 169 & 169 \\
\hline \multirow[t]{4}{*}{$R=\infty$} & $I_{R-\max }(\mu \mathrm{A})$ & 0 & 0 \\
\hline & $V_{R-\max }($ Volt $)$ & 15.77 & 31.6 \\
\hline & $P_{R-\max }(\mu \mathrm{W})$ & 0 & 0 \\
\hline & $z_{\max }(\mu \mathrm{m})$ & 178 & 178 \\
\hline \multirow{4}{*}{$R_{o p t}^{*}=\frac{1}{2 \pi f_{s c} C_{p}}$} & $I_{R-\max }(\mu \mathrm{A})$ & 22.3 & 11.2 \\
\hline & $V_{R-\max }($ Volt $)$ & 5.44 & 10.71 \\
\hline & $P_{R-\max }(\mu \mathrm{W})$ & 60.6 & 60.2 \\
\hline & $z_{\max }(\mu \mathrm{m})$ & 71 & 70.4 \\
\hline
\end{tabular}




\section{List of Figures}

Figure 1 Modelled geometry of piezoelectric cantilever beam with a seismic mass.

Figure 2 Configurations with (a) series connection of piezoelectric layers and (b) parallel connection of piezoelectric layers.

Figure 3 Developed CPC-FEM model of the cantilever beam of piezoelectric layers that are directly connected with a resistive load.

Figure 4 Simulated frequency responses of piezoelectric EHDs of parallel connection of piezoelectric layers for the case of short circuit $(R=0)$, open circuit $(R=\infty)$ and circuit connected with a resistance $(\mathrm{R}=234.2 \mathrm{k} \Omega)$; (a) electric currents through and electric voltages across a resistor and (b) dissipated powers by the resistor and tip vibration displacement amplitudes.

Figure 5 Simulated frequency responses of piezoelectric EHDs of series connection of piezoelectric layers for the case of short circuit $(R=0)$, open circuit $(R=\infty)$ and a resistance ( $R=936.8 \mathrm{k} \Omega$ ); (a) electric currents through and electric voltages across a resistor and (b) dissipated powers by the resistor and tip vibration displacement amplitudes.

Figure 6 Electric and mechanical characteristics versus electric resistance connected to piezoelectric EHDs of parallel connection of piezoelectric layers; (a) electric currents through and voltages across a resistor, (b)dissipated powers by a resistor and tip vibrational displacement amplitudes, and (c) resonant frequency shift of the beam.

Figure 7 (a) Relation of voltage versus current, and (b) relation of dissipated powers by a resistor versus tip vibrational displacement amplitudes of piezoelectric EHDs of parallel connection of piezoelectric layers by use of data shown in Figure 6. 


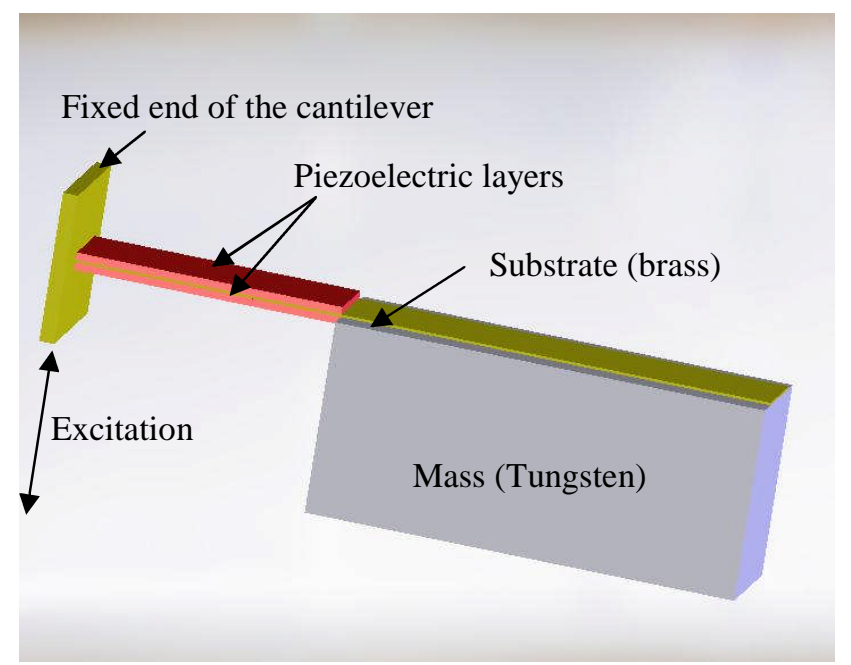

Figure 1 Modelled geometry of piezoelectric cantilever beam with a seismic mass 


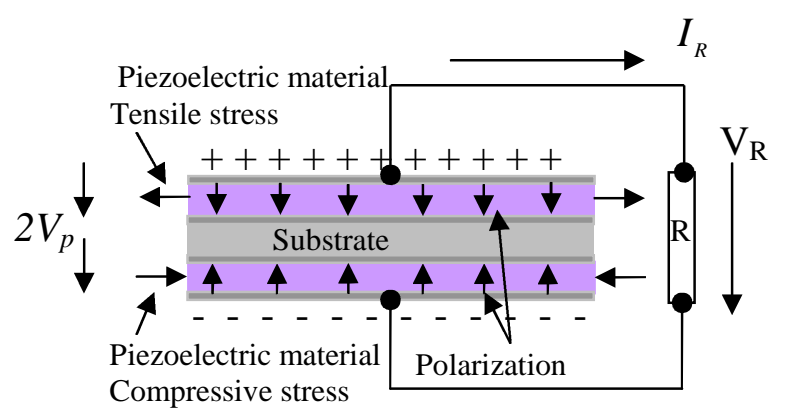

(a)

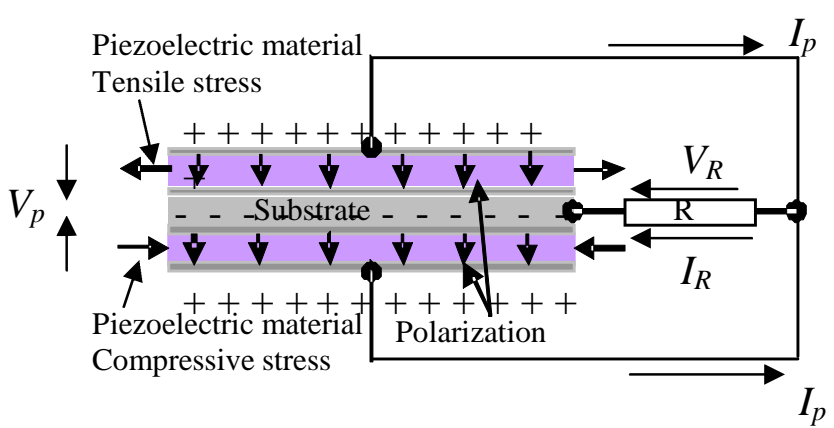

(b)

Figure 2 Configurations with (a) series connection of piezoelectric layers and (b) parallel connection of piezoelectric layers, where "+" represents the plus charges and "-" the minus charge. 


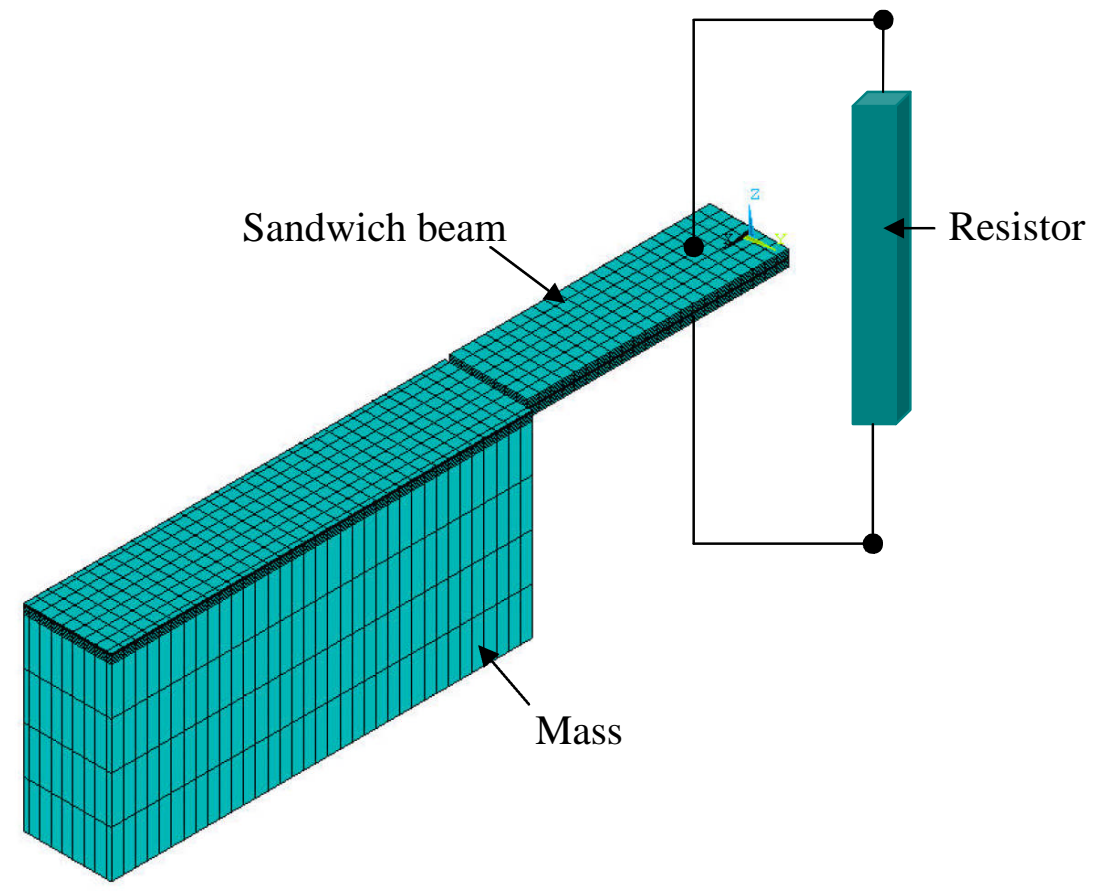

Figure 3 Developed CPC-FEM model of the cantilever beam of piezoelectric layers that are directly connected with a resistive load. 


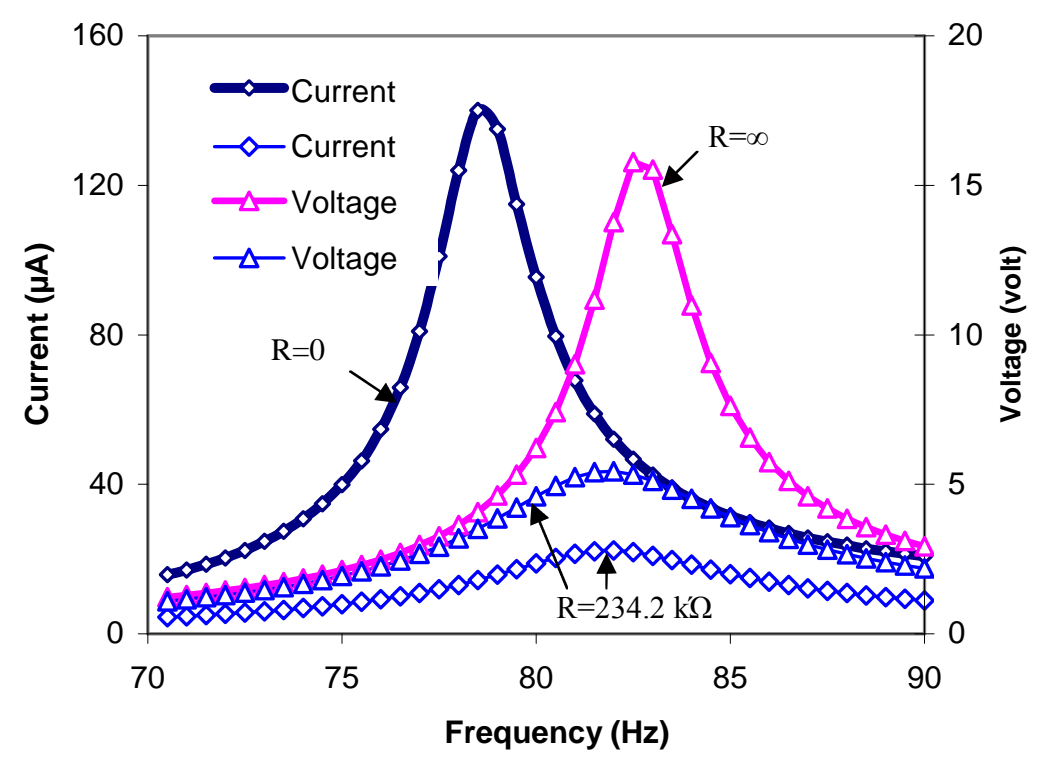

(a)

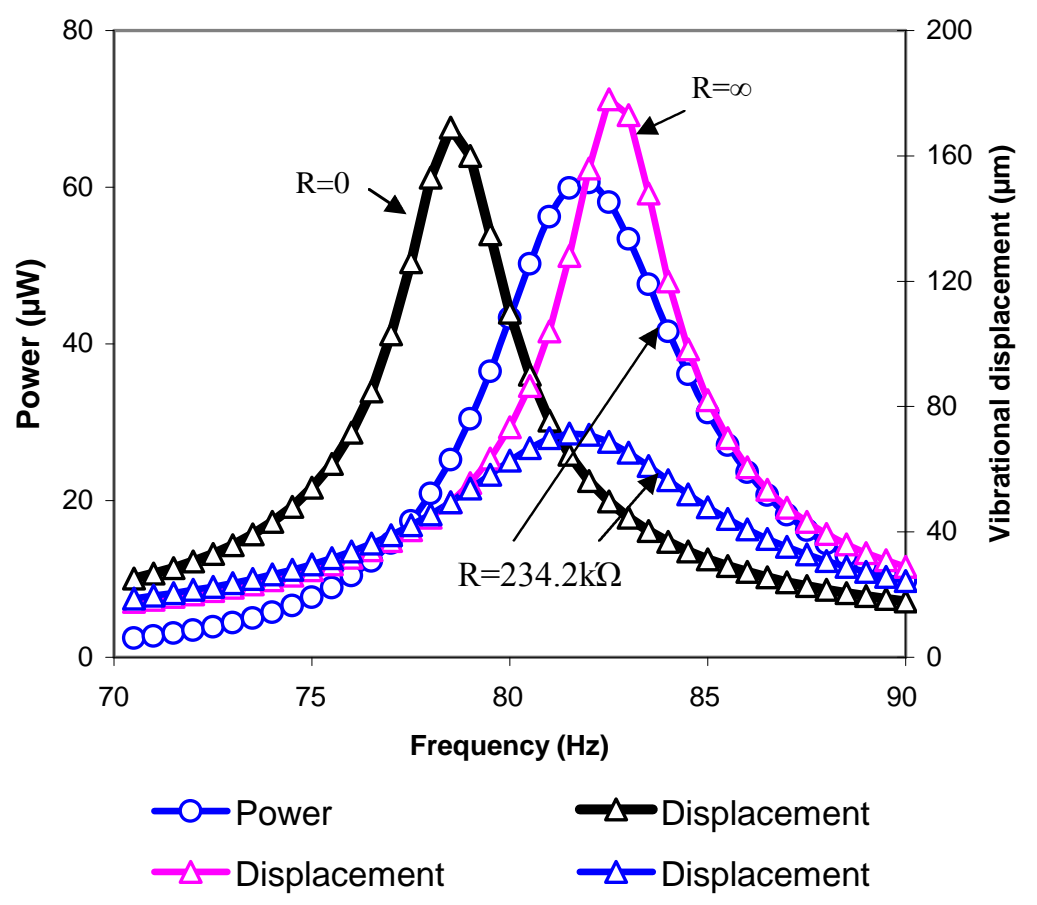

(b)

Figure 4 Simulated frequency responses of piezoelectric EHDs of parallel connection of piezoelectric layers for the case of short circuit $(R=0)$, open circuit $(R=\infty)$ and circuit connected with a resistance $(\mathrm{R}=234.2 \mathrm{k} \Omega)$; (a) electric currents through and electric voltages across a resistor and (b) dissipated powers by the resistor and tip vibration displacement amplitudes. 


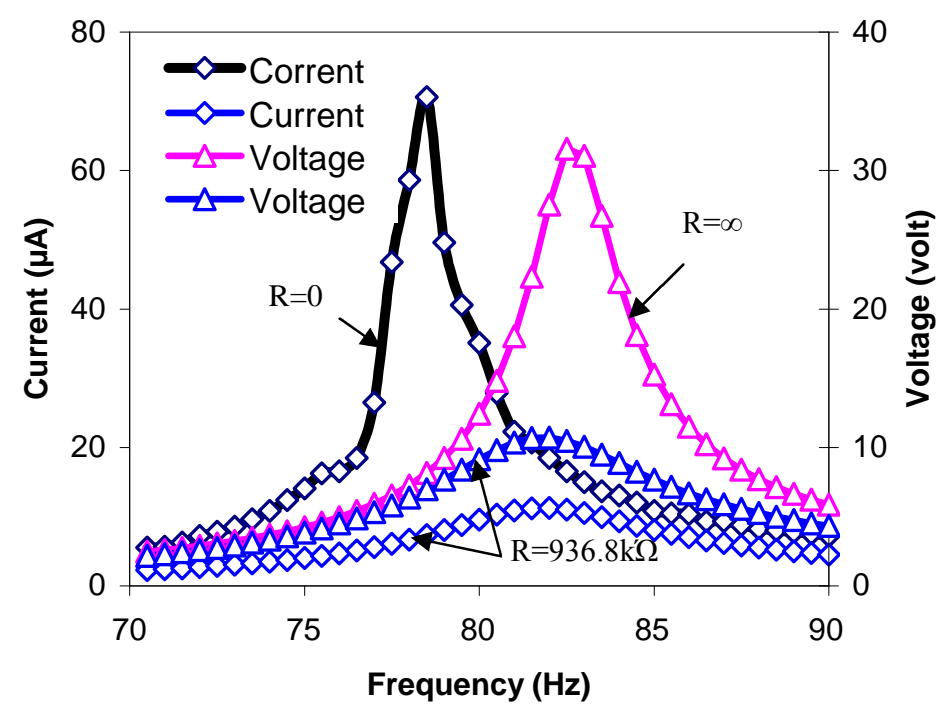

(a)

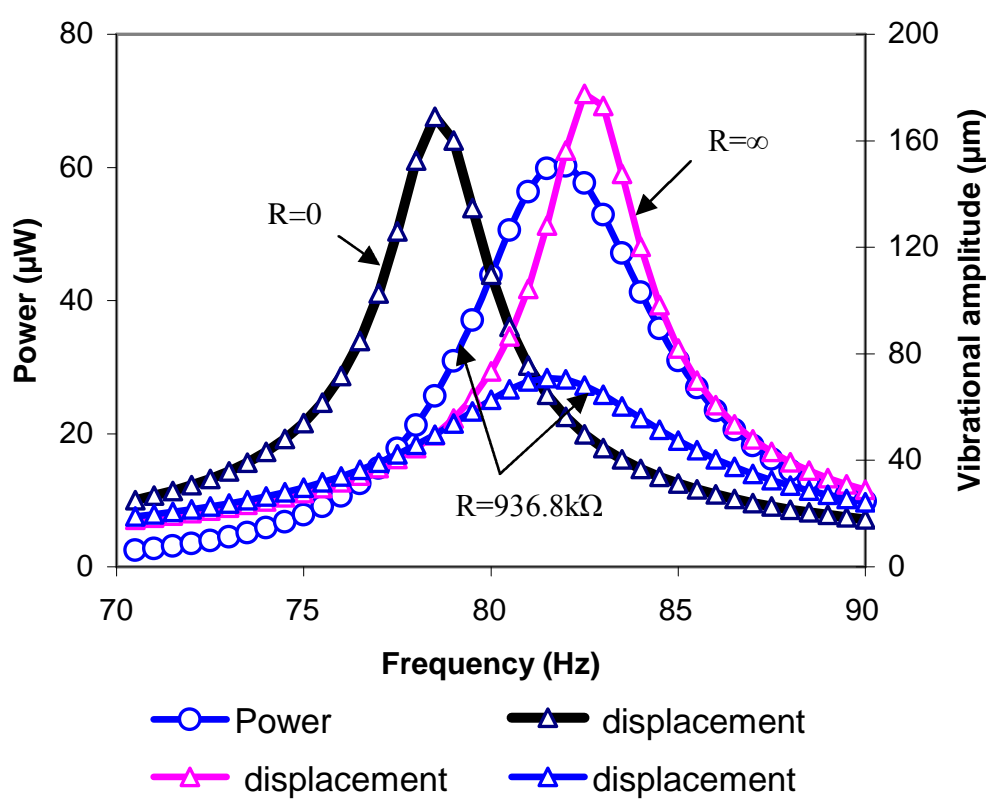

(b)

Figure 5 Simulated frequency responses of piezoelectric EHDs of series connection of piezoelectric layers for the case of short circuit $(R=0)$, open circuit $(R=\infty)$ and a resistance ( $R=936.8 \mathrm{k} \Omega$ ) ; (a) electric currents through and electric voltages across a resistor and (b) dissipated powers by the resistor and tip vibration displacement amplitudes. 


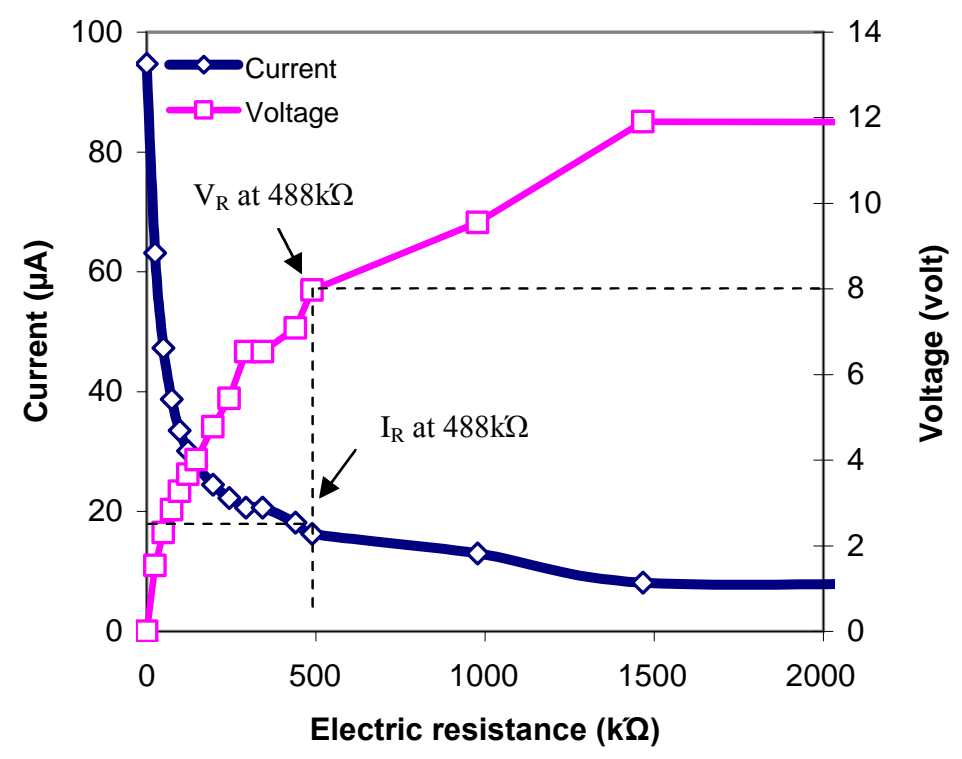

(a)

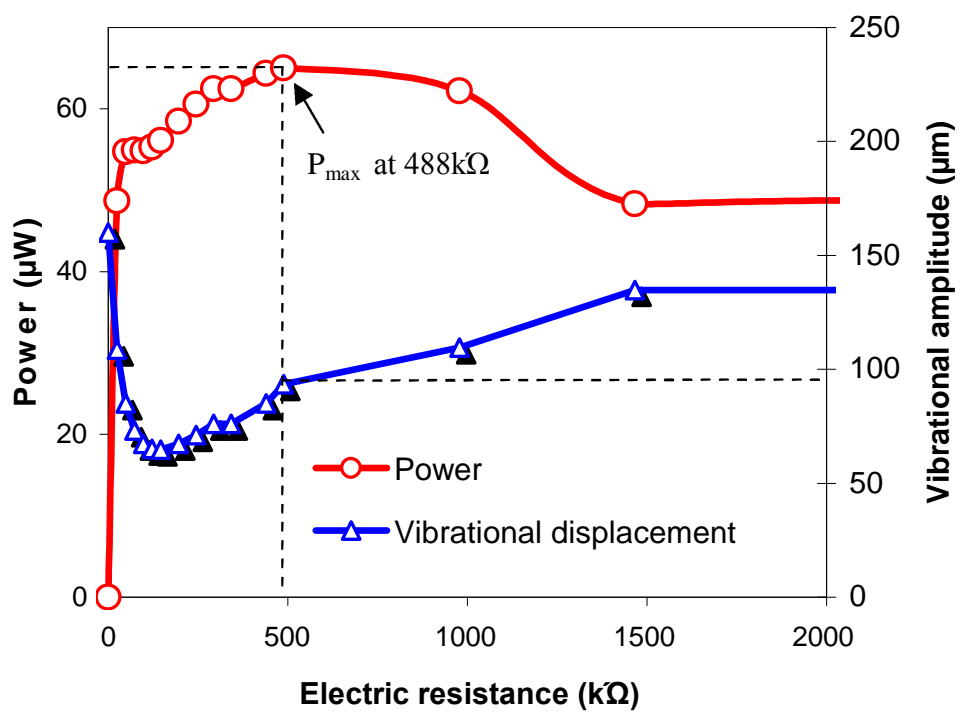

(b) 


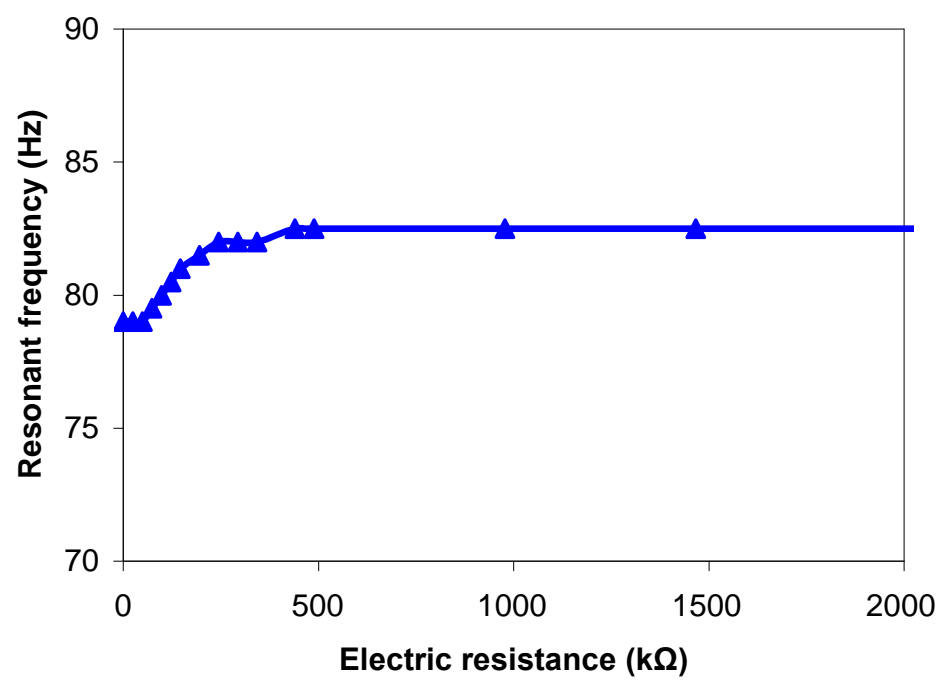

(c)

Figure 6 Electric and mechanical characteristics versus electric resistance connected to piezoelectric EHDs of parallel connection of piezoelectric layers; (a) electric currents through and voltages across a resistor, (b) dissipated powers by a resistor and tip vibrational displacement amplitudes and (c) resonant frequency shift of the beam. 


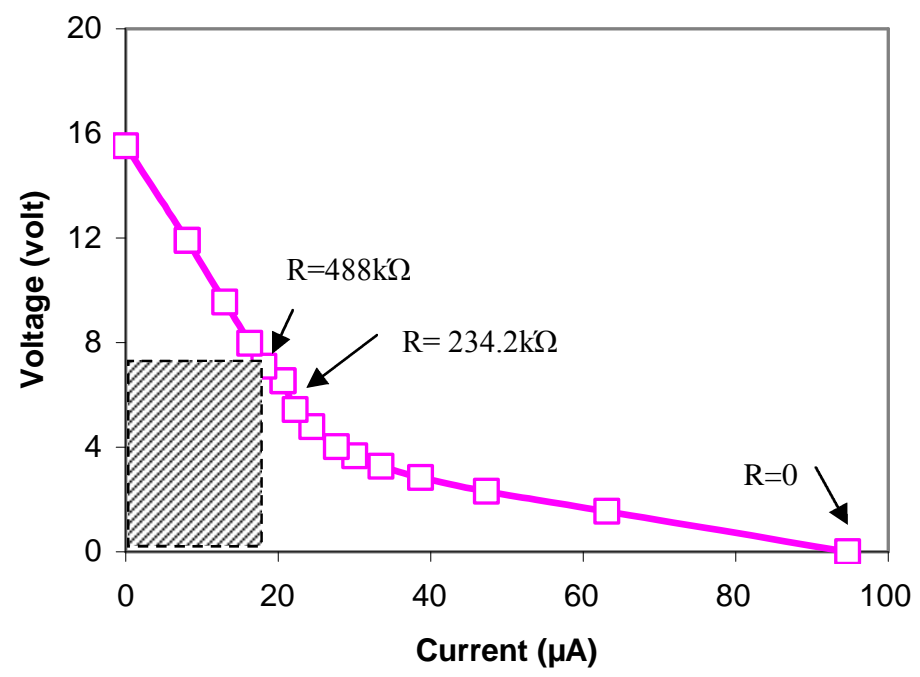

(a)

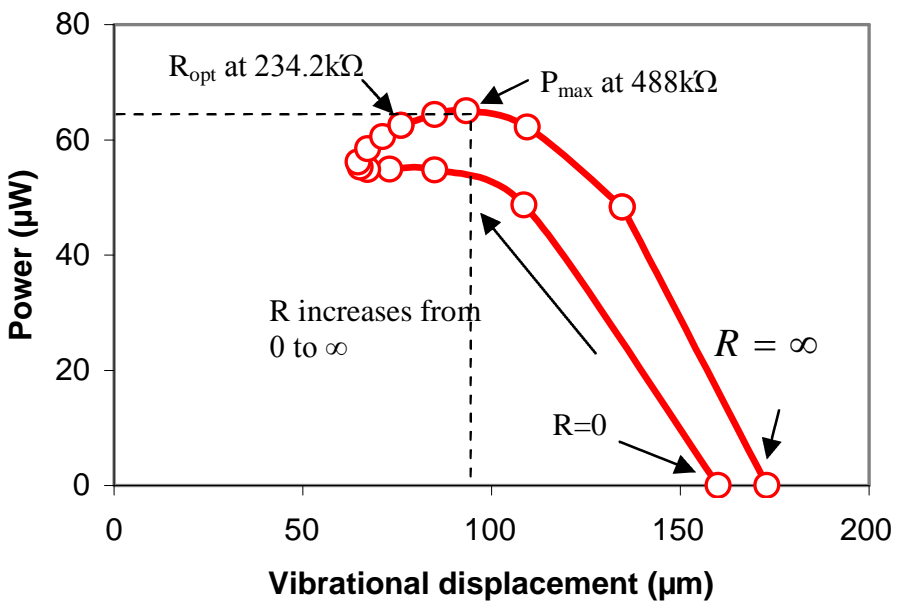

(b)

Figure 7 (a) Relation of voltage versus current and (b) relation of dissipated powers by a resistor versus tip vibrational displacement amplitudes of piezoelectric EHDs of parallel connection of piezoelectric layers by use of data shown in Figure 6. 
Dr. Meiling Zhu gained her BEng degree in 1989, MEng in 1992, and $\mathrm{PhD}$ in 1995 at Southeast University, Nanjing, China. She joined Cranfield University in 2002 as a Research Officer and then became a Senior Research Fellow in 2007. Prior to joining Cranfield she was a Research Fellow at the Biomechanical Research Group, in the University of Leeds (2001-2); an awarded prestigious Research Fellowship of the Alexander von Humboldt at the Institut B für Mechanik, in Stuttgart Universität (1999-2001); a Visiting Scholar at the Mechanical Engineering Department, in the Hong Kong University of Science and Technology (1998-9); and a Post doctor (1995-6) and then an Associate Professor (1996-8) at the Institute of Vibration Engineering Research, in the Nanjing University of Aeronautics \& Astronautics.

Her areas of expertise are design and prototype, modelling (analytical and numerical) and dynamic analytical technology, and characterisation of macro/micro-devices. Her current research is concentrated in piezoelectric energy harvesting devices and novel emerging microdevices and their applications, particularly on design and modelling of devices for design optimisation and then prototype and characterisation of them for applications in wireless communication and medical diagnosis. She has published about 50 refereed journal and conference papers, about two thirds of which are journal papers. She is a Fellow of the Alexander von Humboldt, member of the Institute of Physics, and Chartered Engineer and Scientist. 


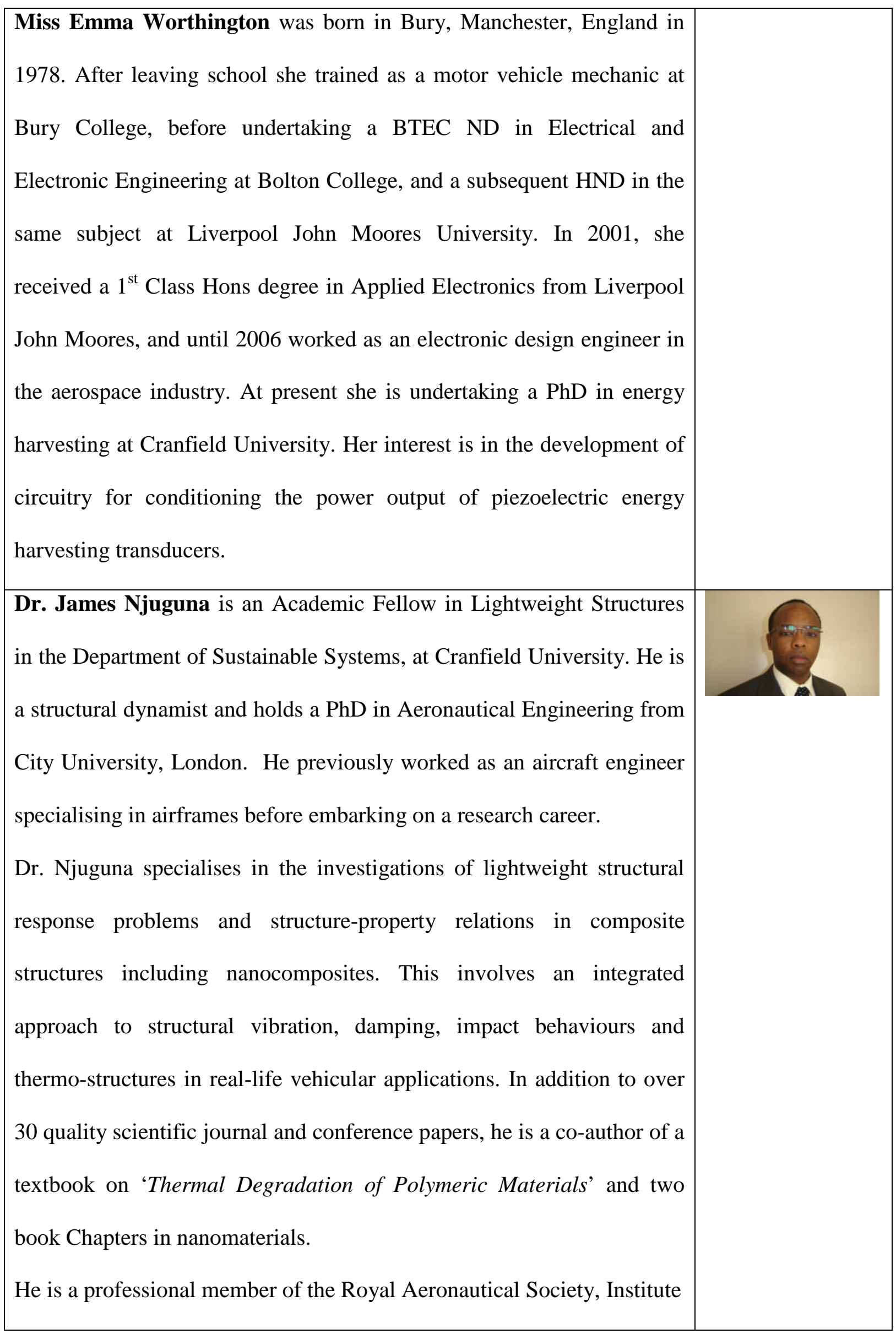


of Nanotechnology, American Institute of Aeronautics and Astronautics.

He is also committee member of Innovations Working Group in the UK Government sponsored Low Carbon Vehicle Partnership (LowCVP). 